\title{
EL SISTEMA GENERAL DE SEGURIDAD SOCIAL EN SALUD DE COLOMBIA. UNIVERSAL, PERO INEFICIENTE: A PROPÓSITO DE LOS VEINTICINCO AÑOS DE SU CREACIÓN*
}

\author{
THE GENERAL SYSTEM OF SOCIAL SECURITY IN HEALTH OF \\ COLOMBIA. UNIVERSAL, BUT INEFFICIENT: A PURPOSE OF \\ TWENTY-FIVE YEARS OF ITS CREATION
}

LE SYSTÈME GÉNÉRAL DE SÉCURITÉ SOCIALE EN SANTÉ DE LA COLOMBIE. UNIVERSEL, MAIS INEFFICIENT: UN OBJECTIF DE VINGT CINQ ANS DE CRÉATION

\section{David MENDIETA GONZÁLEZ** \\ Carmen Elena JARAMILLO ${ }^{* * *}$}

\begin{abstract}
RESUMEN: Según la Constitución colombiana, en su artículo 49, los principios de la seguridad social son la eficiencia, la universalidad y la solidaridad. En 1993, el Congreso de la República expidió la ley 100, que es la base para la prestación de este derecho en general y la salud en particular. En vienticinco años se logró que todos los colombinos fueran cubiertos por el sistema, pero son muchas las voces que denuncian su crisis. Haciendo uso de una metodología descriptivo-analítica, se pudo concluir que hemos alcanzado la universalidad, pero tenemos un sistema ineficiente. Las fuentes utilizadas para apoyar el trabajo son normas jurídicas, doctrina y jurisprudencia.
\end{abstract}

* Recibido el 27 de febrero de 2019 y aceptado para su publicación el 25 de marzo de 2019.

** Abogado, especialista en derecho constitucional y magíster en derecho en la Universidad de Antioquia. Diploma de Estudios Avanzados (DEA) y doctor en derecho constitucional en la Universidad Complutense de Madrid. Director del doctorado en Derecho de la Universidad de Medellín, miembro del Grupo de Investigaciones Jurídicas de la Facultad de Derecho de la Universidad de Medellín. Medellín-Colombia, correo electrónico: dmendieta@ udem.edu.co.

*** Abogada litigante en temas de salud; magíster en derecho en la Universidad de Medellín. Doctoranda en derecho de la misma universidad. Medellín-Colombia, correo electrónico: carmenelenaj@hotmail.com

Revista Latinoamericana de Derecho Social
Núm. 29, julio-diciembre de 2019, pp. 201-218 
Palabras clave: salud, derecho humano, seguridad social, Colombia.

ABSTRACT: According to the Colombian Constitution in its Article 49 the principles of Social Security are efficiency, universality and solidarity. In 1993, the Congress of the Republic issued Law 100, which is the basis for the provision of this right in general and health in particular. In 25 years, all Colombians were covered by the system, but there are many voices that denounce their crisis. Using a descriptive-analytical methodology it was possible to conclude that we have reached universality, but we have an inefficient system. The sources used are legal norms, doctrine and jurisprudence.

Key words: health, human rights, social security, Colombia.

RÉSuMÉ: En vertu de l'article 49 de la Constitution colombienne, les principes de la sécurité sociale sont l'efficacité, l'universalité et la solidarité. En 1993, le Congrès de la République a promulgué la loi 100, qui est à la base de l'exercice de ce droit en général et de la santé en particulier. En 25 ans, tous les Colombiens ont été couverts par le système, mais de nombreuses voix dénoncent leur crise. En utilisant une méthodologie d'analyse descriptive, il a été possible de conclure que nous avons atteint l'universalité, mais notre système est inefficace. Les sources utilisées sont les normes juridiques, la doctrine et la jurisprudence.

Mots-clés: santé, droit humain, sécurité sociale, Colombie.

SUMARIO: I. Introducción. II. Funcionamiento del Sistema General en Seguridad Social en Salud de Colombia. III. Financiamiento del Sistema General de Seguridad Social en Salud de Colombia. IV. La crisis del Sistema General de Seguridad Social en Salud de Colombia. V. La búsqueda de la universalidad del Sistema de Seguridad Social en Salud de Colombia. VI. Conclusiones.

\section{INTRODUCCIÓN}

\section{$\square$} n el contexto internacional existe una preocupación por aumentar el número de personas que tienen acceso a la seguridad en el mundo, independientemente de si se trata de trabajadores formales o informales, de ahí que muchos instrumentos del derecho internacional vayan encaminados a consagrar y materializar la universalidad como principio rector. 
...se reconocen los esfuerzos de cada uno de los instrumentos internacionales por ampliar la cobertura mundial, como la Declaración Universal de Derechos Humanos, el PIDESC, los convenios de la OIT (en especial el 102, de norma mínima de seguridad social), que tienden a reconocer la seguridad social como un derecho humano, con cobertura universal, independiente de las características de formalidad o informalidad laborales. ${ }^{1}$

En este mismo sentido, el artículo 48 de la Constitución colombiana se refiere a la seguridad social como un servicio público de carácter obligatorio que se prestará bajo el control del Estado, bajo los principios de universalidad, eficiencia y solidaridad, y es enfático al afirmar que no se podrán destinar ni utilizar recursos de las instituciones de la seguridad social para fines diferentes a ella.

En el país suramericano, el derecho a la salud se encuentra consagrado en los artículos 44 y 49 del texto constitucional, y hace parte del capítulo de los llamados "Derechos sociales, cultuales y económicos", exceptuando la salud de los niños, que tiene desde 1991 el rango de fundamental. A principio de los noventa se pensó en la salud como el típico derecho de segunda generación, que sólo se podía prestar acorde a las capacidades económicas del Estado. Pero con el paso del tiempo y por vía jurisprudencial se le dio el trato de fundamental, primero, por conexidad con derechos catalogados como tal, y luego, la Corte Constitucional le otorgó el estatus de derecho fundamental autónomo. Lo anterior, hasta que el propio legislador, a través de la ley estatutaria 1751 de 2015, ${ }^{2}$ expresamente reconoció la fundamentalidad de este derecho. ${ }^{3}$

En Colombia, el modelo de seguridad social se creó de forma general en la Ley 100 de 1993, que establece y desarrolla los fundamentos que rigen los sistemas de salud, pensiones y riesgos profesionales. Para tal efecto, la norma trae un numeroso articulado que establece la organización de los sistemas, su dirección y funcionamiento, así como las normas administrativas y financieras, el control y las obligaciones que se derivan de su aplicación. Señala la ley que dentro de los fundamentos del servicio público de la salud se encuentran

1 Mendizábal, G., "Apuntes sobre el derecho internacional de la seguridad social y su relación con américa latina", Revista Latinoamericana de Derecho Social, núm. 25, julio-diciembre de 2017, pp. 37-70.

2 En Colombia, las leyes estatutarias tienen control de constitucionalidad previo. Véase Mendieta, David, "El (des)control de constitucionalidad en Colombia", Estudios Constitucionales, año 16, núm. 2, 2018, pp. 51-88.

3 "La presente ley tiene por objeto garantizar el derecho fundamental a la salud, regularlo y establecer sus mecanismos de protección”, artículo 1o., Ley 1751 de 2015. 
los principios de eficacia, universalidad, solidaridad, integralidad, unidad y participación. ${ }^{4}$

En materia de salud, la ley consagra de manera coordinada una serie de organizaciones, normas y procedimientos de acceso a la comunidad, en general, con el fin de garantizar este derecho, conformando un complejo conjunto de entidades aseguradoras y prestadoras del servicio, entrelazadas, pertenecientes a los sectores público y privado, y que reciben el nombre de Sistema General de Seguridad Social en Salud ( SGSSS).

Durante mucho tiempo, el acceso a la salud para la mayoría de los colombianos se determinó por la capacidad económica de cada quien, y muchos dependían de lo que se les pudiera ofrecer por caridad. Para la época de la expedición de la ley 100 de 1993, sólo un 23.7\% de los colombianos tenían acceso asegurado a la salud y $76.3 \%$ no tenían garantizado el derecho. ${ }^{5}$ Se creó entonces, un sistema donde el Estado y quienes tienen recursos deben subsidiar a los que no los tienen. Para que funcionara el sistema se requería que los primeros fueran cada vez más y los segundos cada vez menos. Es decir, que cada vez más personas pudieran contribuir al pago de su propia salud, y que menos requieran asistencia del Estado.

Aunque es indiscutible el avance en la cobertura de la población, desde hace muchos años se escuchan voces de inconformidad con el sistema, dilación en la prestación del servicio, crisis económica, entre otros. El presente trabajo da fe de algunos cambios importantes que ha tenido el SGSSS durante sus 25 años de existencia en Colombia, abordando cuatro aspectos: 1. Funcionamiento del sistema; 2. Financiamiento del sistema; 3. Crisis del sistema, y 4. Universalidad del sistema, y al final se procederá con unas conclusiones.

\section{FunCiONAMIENTO DEL Sistema GENERAL EN SEguridad Social en SALud EN Colombia}

En Colombia, el derecho a la salud busca garantizar con un sistema de aseguramiento compuesto por el Estado colombiano (Ministerio de Salud y Protección Social [MSPS], la Superintendencia de Salud, los entes territoriales tales como departamentos y municipios), las aseguradoras, que se denomi-

\footnotetext{
4 Artículo 2, Ley 100 de 1993

5 Agudelo, Carlos et al., "El sistema de salud en Colombia: 20 años de logros y problemas", Ciência \& Saúde Coletiva, 16(6), 2011, pp. 2817-2828.
} 
nan Entidades Administradoras de Planes de Beneficios en Salud ${ }^{6}$ (EAPB) (dentro de las cuales están las Empresas Promotoras de Salud [EPS] y las Instituciones Prestadoras del Servicio [IPS]. El SGSSS está divido en tres componentes, a saber: el Régimen Contributivo (RC) para los que tienen capacidad de pago, el Régimen Subsidiado (RS) para los que no tienen capacidad de pago y los Regímenes Especiales (RE) para los empleados de algunas entidades del Estado. ${ }^{7}$

Adicionalmente, son atendidas por el SGSSS personas que lo hacen en forma transitoria mientras se logra su vinculación. Son aquellas que no cuentan con los recursos necesarios para pagar por su propia salud, no hacen parte del RS, pero son atendidos por el sistema; hoy en Colombia son cerca del 5\% de la población total del país, y según datos del Departamento Administrativo Nacional de Estadística (DANE) para 2017 suman más de dos millones de colombianos.

En 1993, cuando se crearon los RC y RS, se establecieron diferencias entre el uno y el otro, no sólo porque el primero funcionaría con los aportes de los afiliados, y el segundo, con el subsidio del Estado y los cotizantes al RC, sino porque el plan ofrecido por el RC fue más amplio en comparación con lo que ofrecía el RS, estableciendo un trato diferencial en materia de salud en Colombia, algo así como personas de primera clase y personas de segunda clase, en un país marcado por las estratificaciones. Esto cambió con la expedición por parte de la Corte Constitucional, de la Sentencia T 760 de 2008, que ordenó unificar ambos planes, lo que para muchos significó un triunfo para la dignidad de los colombianos. ${ }^{8}$

"En lo que respecta a los planes de beneficios, se impartirán ocho órdenes. A saber,... (6) ordenar que se unifique el POS de los menores de edad; (7) ordenar que se adopte un programa y un cronograma para unificar el POS en el caso de las demás personas...".?

6 Ministerio de Salud y Protección Social, Dirección de Epidemiología y Demografía, Guía conceptual y metodológica para la caracterización de la población afiliada a las Entidades Administradoras de Planes de Beneficios de Salud (EAPB), Bogotá, 2017.

7 Ocampo, J. y Osley Garzón, M., "El sistema de riesgos laborales frente al trabajador del sector informal”, Opinión Jurídica, 15 (30), pp. 183-204, 15 de diciembre de 2016, disponible en: https:// doi.org/ https:// doi.org/10.22395/ojum.v15n30a9.

8 Mendieta, D. y Tobón, M. L. (2018a), "La dignidad humana y el Estado social y democrático de derecho: el caso colombiano", Revista de Estudos Constitucionais, Hermenêtica e Teoria do Direito (RECHTD), 10(3):95-108, septiembre-diciembre de 2018.

9 Corte Constitucional de Colombia, Sentencia T 760 de 2008. 
Con la expedición de la Ley estatutaria 1751 de 2015, el Plan Obligatorio de Salud (POS) desapareció, y se establece que los usuarios del SGSSS tienen derecho a todos los procedimientos y medicamentos que sean necesarios para prevenir y enfrentar una enfermedad, excepto aquellos que están expresamente excluidos. La ley le concedió al MSPS dos años para la creación de un mecanismo técnico científico, público, colectivo, participativo y transparente de exclusión de servicios o tecnologías de la financiación con dineros del SGSSS. ${ }^{10}$

En febrero de 2017, cuando se cumplieron los dos años dados por el legislador para la entrada en vigencia de esta condición, el MSPS expidió la resolución 0330, en la cual se fija un sistema de exclusión a cargo de la Dirección de Regulación de Beneficios, Costos y Tarifas del Aseguramiento en Salud, ente adscrito al MSPS, y se estableció que el proceso de exclusión deberá cumplir con las siguientes fases:

- Nominación y priorización.

- Análisis técnico-científico.

- Consulta a pacientes potencialmente afectados.

- Adopción y publicación de decisiones. ${ }^{11}$

Mediante la resolución 5267 de 2017 del MSPS se excluyeron algunos medicamentos, pero también servicios y productos, como colegios e instituciones educativas, educación especial, toallas higiénicas, pañitos húmedos, papel higiénico, entre otros. ${ }^{12}$ Lo que llevará a quienes requieran alguno de estos medicamentos o productos excluidos a acudir a la tutela que en Colombia es el mecanismo rápido y efectivo para reclamar ante los jueces la protección de los derechos fundamentales.

Inicialmente, el POS cubría sólo las enfermedades generales y algunas denominadas graves o congénitas, pero con la ayuda de la jurisprudencia de la Corte Constitucional colombiana, que impulsó la reforma del SGSSS, se ha ido logrando progresivamente la atención de enfermedades y procedimientos médicos que pueden ser costosos para el sistema.

En Colombia, la vulnerabilidad de la población se mide con un sistema de puntos que determina el acceso a los programas de asistencia social del

\footnotetext{
10 Ley 1751, artículo 15, parágrafo 10.

11 Resolución 0330 de 2017, del Ministerio de Salud y Protección Social, artículos 8 y ss.

12 Resolución 5267 de 20171 del Ministerio de Salud y Protección Social, anexo técnico.
} 
Estado, denominado SISBEN..$^{13}$ Para ser parte del RS se requiere estar inscrito en este sistema en los niveles 1 y 2, lo que significa estar censado como población pobre o que requiere atención prioritaria (niños abandonados o afectados por el conflicto, desplazados, indígenas, inmigrantes, como es el caso de los venezolanos, entre otros).

El decreto 1011 de 2006 les exige a los prestadores del servicio, sean del sector público o del privado, a constituirse como IPS e inscribirse en el Registro Especial de Prestadores de Servicios en Salud (REPSS). Se estima que para 2018 existan en Colombia 11.500 de ellas. Estas instituciones prestan el servicio según tres niveles de complejidad. ${ }^{14}$ Los tratamientos más complejos, pero también más costosos, son realizados por las del tercer nivel. Esto ha llevado a que las IPS públicas que deberían tener vocación de servicio a todos los colombianos, en la práctica hayan descuidado la atención en el primer y segundo nivel, y enfoquen sus esfuerzos humanos, económicos y tecnológicos para la atención en el tercero.

Con respecto a los prestadores públicos, se observa una disminución en la oferta de servicios de los primeros niveles de atención, a partir de la información del Registro Especial de Prestadores de Servicios de Salud, la cual ha disminuido entre 2013 y 2016 en un $2.8 \%$; en el segundo nivel se presenta el mismo comportamiento con una disminución del 1.5\%; llama la atención el crecimiento significativo de las IPS públicas de tercer nivel de atención las cuales crecen durante el mismo periodo en $9.8 \% .^{15}$

Algunos prestadores han hecho un gran esfuerzo para certificarse como IPS de alta calidad. En julio de 2010 se contaba con diecinueve de estos prestadores acreditados, y a diciembre de 2018 ya había en el país 44 avaladas en estándares de excelencia. ${ }^{16}$ Éste es uno de los aspectos contradictorios de la salud en Colombia, al mismo tiempo que vivimos la mayor crisis de credibilidad del sector ante la sociedad; en 2018, veintitrés de nuestros hospitales están entre los 58 mejores de América Latina. ${ }^{17}$

Es innegable el logro de alcanzar en veinticinco años la cobertura universal en salud para los colombianos (para 2017 representaba el 94.6\% de la pobla-

13 Disponible en: bttps:// www.sisben.gov.co/sisben/paginas/que-es.aspx.

14 Resolucion 5261 de 1994.

15 Ministerio de Salud y Protección Social, Politica de atención integral en salud, Bogotá, 2016.

16 Disponible en: http:// wmw.acreditacionensalud.org.co/Documents/IPSacreditadas.pdf.

17 Disponible en: https:// clustersalud.americaeconomia.com/gestion-hospitalaria/ ranking-de-clinicas -y-hospitales-2018-estos-son-los-mejores-de-latinoamerica. 
ción asegurada, y el 5.2\% faltante también tiene derecho a ser asistido). Pero no todos los colombianos tienen acceso efectivo a la misma calidad del servicio, pues existen muchas variables, tales como lugar de residencia, ${ }^{18}$ si la EPS a la que se pertenece tiene o no contrato vigente con las IPS que poseen mejor experticia y tecnología, si la EPS tiene o no créditos con las IPS, lo que significa que estamos frente a un sistema que de nuevo estratifica a los colombianos.

...En economías con fuertes disparidades distributivas, el problema más importante es la tendencia natural que genera el mercado a que el sector privado oriente su oferta — en cantidad y especialmente en calidad — hacia los sectores de mayores ingresos. Este problema no se soluciona necesariamente con un esquema de subsidios a la demanda y exige, por lo tanto, que el Estado diseñe instrumentos para aumentar la oferta dirigida a los sectores de bajos ingresos. ${ }^{19}$

\section{FinANCIAMIENTO DEL Sistema GENERAL DE SEguridad Social EN SALUd EN COLOMBia}

El sistema tiene diferentes fuentes de financiamiento. En primer lugar, los aportes de los trabajadores asalariados y sus empleadores, los independientes con capacidad de pago y los pensionados, los cuales, en los tres casos se afilian a una EPS, y, en segundo lugar, con contribuciones del Estado. Todos estos recursos se reúnen en la Administradora de los Recursos del Sistema General de Seguridad Social en Salud (Adres), que los redistribuye a las EPS, según el número de afiliados que tienen en cada uno de los dos regímenes. En el país, el $50 \%$ de la población cubre con sus aportes y cotizaciones cerca del $70 \%$ de los gastos del sistema, y el otro 30\% es asumido por el Estado. "En Colombia, los recursos financieros que se obtienen por medio de cotizaciones y aportes representan cerca del $70 \%$ del total, mientras que más del 50\% de los afiliados se encuentran en el régimen subsidiado". ${ }^{20}$

18 Duque Quintero, S. et al., "La seguridad social como un derecho fundamental para las comunidades rurales en Colombia”, Opinión Jurídica, 22 de enero de 2018, 16(32), pp. 189-209, disponible en: https:// doi.org/ https:// doi.org/10.22395/ojum.v16n32a8.

19 Ocampo, José Antonio, "Distribución del ingreso, pobreza y gasto social en América Latina”, Revista de la CEPAL, núm. 65, agosto de 1998.

20 Agudelo, Carlos et al., "El sistema de salud en Colombia: 20 años de logros y problemas", Ciência \& Saúde Coletiva, 16(6), 2011, pp. 2817-2828. 
Es importante aclarar que el artículo 66 de la ley 1753 de 2015, por medio de la cual se expide el Plan de Desarrollo 2014-2018, crea la entidad Adres, con el fin de asegurar la optimización de los recursos del SGSSS y realizar los adecuados controles. La nueva entidad está adscrita al MSPS, cuenta con personería jurídica, autonomía administrativa y financiera y patrimonio independiente. La entidad descrita remplazó al Fondo de Seguridad y Garantía (Fosyga).

El Adres es una entidad descentralizada del orden nacional, asimilable a una empresa industrial y comercial del Estado, con personería jurídica, autonomía administrativa y financiera y patrimonio independiente. Su propósito es administrar todos los recursos del SGSSS. Cuenta con un director general y una junta; el primero, nombrado por el presidente de la República, de dedicación exclusiva y de libre nombramiento y remoción, y la segunda, integrada por el ministro de Salud (quien la preside), el ministro de Hacienda, el director de planeación nacional, un representante de los gobernadores y un representante de los distritos y municipios.

Como se dijo antes, en el RC se afilian las personas que tienen como hacer aportes para su propia salud (trabajadores del sector público y privado, pensionados, independientes). Este sistema opera con base en una cotización de sus afiliados, más los aportes de los empleadores. El RC se financia con los aportes de los trabajadores, cuya contribución es obligatoria y equivale a $12.5 \%$ de los ingresos laborales o salario base de cotización, de los cuales al trabajador se le descuenta un $4 \%$ de su salario, mientras que el empleador se encarga de pagar el restante $8.5 \%{ }^{21}$ Los trabajadores independientes y los pensionados deben pagar la totalidad de la contribución.

El RS se encarga de asegurar a las personas con menos recursos y que se encuentran identificadas por el Sisben. El RS se financia con 1.5\% que apor$\tan$ los trabajadores, el 1\% que dan los pensionados y otros actores del RC, ${ }^{22}$ recursos de las entidades territoriales y demás fuentes establecidas en la ley.23

Los RE afilian a los trabajadores de las fuerzas militares, la policía nacional, la Empresa Colombiana de Petróleos (Ecopetrol), los docentes que laboran para el Estado y las universidades públicas. Los RE operan con recursos de las cotizaciones de los funcionarios públicos más los aportes del Estado. Para los fines del presente trabajo, los RE serán tenidos en cuenta como parte

\footnotetext{
21 Ley 1122 de 2007, artículo 10.

22 Idem.

23 Ibidem, artículo 11.
} 
Esta revista forma parte del acervo de la Biblioteca Jurídica Virtual del Instituto de Investigaciones Jurídicas de la UNAM

del RC. Los recursos públicos con destinación a la salud son inembargables, tienen destinación específica y no se les podrá dar uso diferente al que se establece en la Constitución y en la Ley. ${ }^{24}$

\section{LA CRISIS DEL SISTEMA GENERAL DE SEGURIDAD SOCIAL EN SALUD DE COLOMBIA}

Los recursos destinados al sector salud en Colombia son millonarios; representan un porcentaje importante del producto interno bruto (PIB), y cuyo promedio en los últimos vienticinco años es del $6 \%$. El siguiente cuadro presenta una relación del PIB total por cada año, actualizando valores constantes en dólares y el porcentaje dedicado a la salud.

\begin{tabular}{|c|c|c|c|c|c|c|c|}
\hline 1993 & 1994 & 1995 & 1996 & 1997 & 1998 & 1999 & 2000 \\
\hline 166271.7 & 174830.3 & 183925.8 & 187707.0 & 194145.9 & 195252.1 & 187043.7 & 192514.5 \\
\hline 6.2 & 6.8 & 6.76 & 8.16 & 8.96 & 8.73 & 8.67 & 5.91 \\
\hline
\end{tabular}

\begin{tabular}{|c|c|c|c|c|c|c|c|}
\hline 2001 & 2002 & 2003 & 2004 & 2005 & 2006 & 2007 & 2008 \\
\hline 195744.7 & 200646.1 & 208507.9 & 219627.7 & 229964.6 & 245554.8 & 262372.0 & 270917.6 \\
\hline 5.96 & 5.67 & 5.92 & 5.37 & 5.82 & 6.11 & 6.25 & 6.62 \\
\hline
\end{tabular}

\begin{tabular}{|c|c|c|c|c|c|c|}
\hline 2009 & 2010 & 2011 & 2012 & 2013 & 2014 & 2015 \\
\hline 274183.3 & 286103.6 & 307168.1 & 319157.0 & 333732.5 & 349512.4 & $359843.8^{25}$ \\
\hline 7.01 & 6.75 & 6.54 & 6.83 & 6.81 & 6.4 & $6.2^{26}$ \\
\hline
\end{tabular}

24 Ley 1751 de 2015, artículo 25.

25 Producto Interno Bruto (PIB) total anual a precios constantes en dólares, disponible en: http:/ / estadisticas.cepal.org/sisgen/ConsultaIntegrada.asp?IdAplicacion $=6$ \&idTema $=131$ \&idIndica dor $=2204$ \&idioma $=e$.

26 El porcentaje del PIB con destinación a la salud en Colombia fue obtenido con información encontrada en Gilberto Barón-Leguizamón, "Gasto nacional en salud de Colombia 1993-2003. Composición y tendencias”, Revista SaludPública, 9 (2), 2007, pp. 167-179, y la CEPAL, disponible en: http://interwp.cepal.org/sisgen/ConsultaIntegrada.asp?idIndicador $=55$ \&idioma $=e$. Se debe aclarar que para la fecha de construcción del texto no se encontraron datos de los años 2016, 2017 y 2018. 
Para muchos, el SGSSS ha convertido la salud en un negocio, cuya cobertura es universal, pero de mala calidad y excluyente, pues muchas EPS establecen trabas al goce efectivo del derecho por parte de sus usuarios, obligándolos a tener que acudir a la vía judicial para acceder al derecho a través de la acción de tutela (mecanismo de amparo existente en Colombia para garantizar los derechos fundamentales). Para 2018 se presentaron en Colombia un total de 583.000 tutelas, de las cuales 197.055 fueron contra el sector salud; es decir, el 33\%, y según las palabras del presidente de la Corte Constitucional, Alejandro Linares, la crisis de la salud se evidencia por el alto número de tutelas instauras por los colombianos. "Creemos que la situación del Sistema de Salud sigue siendo crítica, eso se manifiesta en el número de tutelas que sigue la gente interponiendo en materia de acceso a la salud en todas sus formas". ${ }^{27}$

Desconocer o entorpecer el acceso al goce efectivo del derecho a la salud por el ánimo de lucro ha llevado a que el SGSSS colombiano esté siendo cuestionado, y muchos lo tildan de fallido y desprestigiado. "El afán por apropiarse de las utilidades económicas de este mercado es, en el fondo, el factor que ha impulsado y mantenido el modelo sanitario de Colombia a pesar de sus evidentes fallos y su desprestigio". 28

Desde hace mucho tiempo, los colombianos sabemos que nuestro SGSSS está en crisis. Es común que los titulares de los periódicos y demás medios de comunicación nos lo recuerden, y no nos hemos repuesto de un escándalo, en el sector, cuando tenemos que enfrentar uno nuevo. Alcanzamos la universalidad, pero los usuarios se quejan de la mala calidad del servicio prestado.

El fenómeno financiero de la integración vertical, el uso indebido de recursos públicos, los sospechosos recobros de las eps al Fosyga e innumerables casos en los que se evidencia la muerte de pacientes debido a la pésima calidad del servicio, son apenas la punta del iceberg de un problema social caracterizado por su crisis en variables estructurales. ${ }^{29}$

27 Alejandro Linares, presidente de la Corte Constitucional de Colombia en entrevista dada a la cadena radal Caracol, el 18 de diciembre de 2018, disponible en: http:/ / caracol.com.co/ radio/2018/12/19/judicial/1545179479_836263.html.

28 Gómez-Arias, R., "Colombia: ¿qué ha pasado con su reforma de salud?”, Revista Peruana de Medicina Experimental y Salud Publica, vol. 31, núm. 4, Lima, octubre-diciembre de 2014.

29 Rivera, A. F., "Coyuntura del sistema de salud en Colombia: caracterización de una crisis, desde las particularidades financieras de las EPS”, Gestión \& Desarrollo, 10 (1), 2013, pp. 103-116. 
El modelo de intermediación de las EPS en Colombia lleva mucho tiempo en entredicho. Recursos de la salud han sido utilizados en actividades ajenas a garantizar el derecho. Frente a la mala prestación del servicio, denuncias por corrupción y la incapacidad económica, varias aseguradoras han tenido que ser intervenidas y liquidadas, dejando deudas multimillonarias a las IPS e, incluso, llevando a su quiebra.

La combinación de crisis de legitimidad, crisis regulatoria generalizada e incapacidad de las aseguradoras en salud para responder por sus obligaciones, fue generando condiciones percibidas por los agentes públicos y privados como una crisis sectorial que afecta las expectativas futuras para el desarrollo del derecho a la salud y la garantía del goce efectivo al acceso, uso y calidad de los servicios... ${ }^{30}$

\section{LA BÚSQUEDA DE LA UNIVERSALIDAD DEL SISTEMA GENERAL DE SEguridad Social en SAlud de Colombia}

Como se dijo en la introducción de este trabajo, la universalidad es uno de los primeros principios enunciados por el artículo 48 de la Constitución colombiana, pero pasados 25 años chemos cumplido con este mandato? Para 2018, Colombia cuenta con 45 millones de habitantes con acceso al SGSSS con sus tres regímenes: el RC, el RS y los RE.

El sistema ha ido cubriendo progresivamente la mayor parte de la población colombiana. En el siguiente cuadro se muestra la evolución de esta cobertura. Se han tomado los años de los que consta la información por parte del Departamento Admnistrativo Nacional de Estadísticas (DANE); así:

\begin{tabular}{|c|c|c|c|c|}
\hline Año & $\begin{array}{c}\text { Porcentaje de la } \\
\text { población total } \\
\text { del país cubierta } \\
\text { por el SGSSS }\end{array}$ & $\begin{array}{c}\text { De los que se encuentran } \\
\text { cubiertos por el SGSSS } \\
\text { que porcentaje pertenece } \\
\text { al RC }\end{array}$ & $\begin{array}{c}\text { De los que se encuentran } \\
\text { cubiertos por el SGSSS } \\
\text { que porcentaje pertenece } \\
\text { al RS }\end{array}$ & $\begin{array}{c}\text { Porcentaje de la } \\
\text { población total del } \\
\text { país no cubierta } \\
\text { por el SGSSS }\end{array}$ \\
\hline 1993 & 23.7 & 22.4 & & 76.3 \\
\hline 1997 & 57.2 & 37.6 & 19.6 & 42.8 \\
\hline
\end{tabular}

30 Suárez-Rozo, Luisa Fernanda et al., "La crisis del sistema de salud colombiano: una aproximación desde la legitimidad y la regulación”, Rev. Gerenc.. Polí. Salud, 2017, 16 (32), pp. 34-50, disponible en: https:// doi.org/10.11144/Javeriana.rgps16-32.cssc. 
Esta revista forma parte del acervo de la Biblioteca Jurídica Virtual del Instituto de Investigaciones Jurídicas de la UNAM http://www.juridicas.unam.mx/

DOI: http://dx.doi.org/10.22201/iij.24487899e.2019.29.13905

\begin{tabular}{|c|c|c|c|c|}
\hline Año & $\begin{array}{c}\text { Porcentaje de la } \\
\text { población total } \\
\text { del país cubierta } \\
\text { por el SGSSS }\end{array}$ & $\begin{array}{c}\text { De los que se encuentran } \\
\text { cubiertos por el SGSSS } \\
\text { que porcentaje pertenece } \\
\text { al RC }\end{array}$ & $\begin{array}{c}\text { De los que se encuentran } \\
\text { cubiertos por el SGSSS } \\
\text { que porcentaje pertenece } \\
\text { al RS }\end{array}$ & $\begin{array}{c}\text { Porcentaje de la } \\
\text { población total del } \\
\text { país no cubierta } \\
\text { por el SGSSS }\end{array}$ \\
\hline 2003 & 61.8 & 38.9 & 22.9 & 38.2 \\
\hline 2008 & 86 & 41.71 & 43.95 & 13.8 \\
\hline 2010 & 88.7 & 42.13 & 46.12 & 11.1 \\
\hline 2011 & 90.1 & 45.14 & 44.78 & 9.7 \\
\hline 2012 & 90.6 & 43.04 & 47.3 & 9.2 \\
\hline 2013 & 91.3 & 44.83 & 46.38 & 8.6 \\
\hline 2014 & 94.1 & 45.07 & 48.83 & 5.8 \\
\hline 2015 & 94.6 & 47.87 & 46.54 & 5.2 \\
\hline 2016 & 95.4 & 47.8 & 47.4 & 4.4 \\
\hline 2017 & 94.6 & 47.02 & 47.3 & 5.2 \\
\hline
\end{tabular}

FUENTE: Elaboración propia con información suministrada por el DANE, excepto el año 1993, pues la información fue obtenida del texto de Carlos Agudelo que consta en las referencias.

Con la información anterior se puede decir que ha habido avances significativos en el número de personas cubiertas por el SGSSS, ya que ha pasado de un $23.7 \%$ en 1993 , hasta llegar a un $94.6 \%$ en 2017 , y logrado la mayor cobertura en 2016, con un $95.4 \%$. Resta un $5.2 \%$ de la población que no está asegurada, pero sí tiene acceso al derecho, lo que nos lleva a concluir que en Colombia hemos alcanzado la universalidad real del derecho a la salud. "Asimismo, la universalización de la atención es un aspecto decisivo para seguir avanzando en la materialización del derecho a la salud. Pero, sigue siendo un reto el logro pleno de la universalidad". ${ }^{31}$

Sin embargo, es importante no equiparar la cobertura universal con la accesibilidad. La gran mayoría de los colombianos pertenecen a un régimen de salud, ya sea contributivo o subsidiado, pero esto no significa que todos tengan acceso efectivo a IPS acreditas por sus estándares de alta calidad y profesionales especialistas en las muchas áreas de la medicina. Es diferente cobertura que accesibilidad.

31 Agudelo, Carlos et al., "El sistema de salud en Colombia: 20 años de logros y problemas", Ciência \& Saúde Coletiva, 16(6), 2011, p. 2825. 
Lo anterior refleja que existe una inequitativa distribución de la oferta de prestadores de servicios de salud en Colombia que impide un acceso equitativo a la atención médica. ${ }^{32}$

Es tan abstracta la accesibilidad a los servicios de salud, que se han generado diversas barreras que impiden que la atención en salud se materialice de una manera efectiva. ${ }^{33}$

Uno de los retos del SGSSS es que el número de personas que son parte del RC sea superior que los que pertenecen al RS. Aunque entre 1993 y 2003 el número de afiliados al RC era superior que los del RS, pero muchos no estaban cubiertos por el SGSSS, entre 76.3\% (1993) y 38.2\% (2003); pero en la medida en que más de estos colombianos ingresan al SGSSS, la brecha entre el RC y el RS desaparece, e incluso la tendencia es que haya más colombianos en el RS que en RC (2008, 2010, 2012, 2013 y 2014), y pocas veces se logró que las personas del RC fueran más que las del RS y por un estrecho margen (2011, 2015 y 2016). "En la medida en que el sector público opera como un «asegurador de última instancia» facilita la selección (discriminación) de riesgos por parte de las aseguradoras privadas, lo que redunda en una menor eficiencia y equidad". 34

Aunque en los primeros cinco años del SGSSS hubo avances importantes en el proceso de formalización de trabajadores, en los últimos veinte años sólo se ha logrado el incremento de diez puntos del número de personas que se encuentran dentro del RC, al pasar del $37.6 \%$ en 1997 , al $47.2 \%$ en 2017 , lo que significa que sólo un 10\% de la población nacional, en margen de tiempo tan amplio (veinte años) pudo adquirir las condiciones para ingresar al RC; es decir, vincularse laboralmente, mediante un contrato de trabajo, ser servidor público, pensionarse o ser trabajador independiente con capacidad de pago. Lo anterior es corroborado al sumar el número de afiliados al RS con los que no hacen parte del sistema en $1997(19.6 \%+42.8 \%=62.4 \%)$ y repetir la operación en $2017(47.3 \%+5.2 \%=52.5 \%)$. De nuevo la diferencia es un $10 \%$, que son los que han ingresado al RC.

32 Ayala, Jhorland, "La salud en Colombia: más cobertura, pero menos acceso", Documentos de Trabajo sobre la Economía Regional, Bogotá, Banco de la República, núm. 204, julio de 2014, p. 27.

33 Álvarez, Gabriel, "Crisis de la salud en Colombia: limitantes del acceso al derecho fundamental a la salud de los adultos mayores", Revista CES Derecho, vol. 7, núm. 2, juliodiciembre de 2016, pp. 106-125.

34 Titelman, D., Ensayos sobre el financiamiento de la seguridad social en salud. Los casos de Estados Unidos-Canadá-Argentina-Chile-Colombia, México, Fondo de Cultura Económica, 2000, p. 47. 
Con las cotizaciones se financia el régimen contributivo. En tanto se trata de un impuesto al trabajo, su magnitud está gobernada en gran parte por el empleo en el sector formal de la economía y los niveles salariales; es debilitada por el desempleo, la informalidad, la evasión y elusión de las cotizaciones. ${ }^{35}$

Que un país, en un plazo de veinte años, haya formalizado sólo el 10\% de su población, pasando de $37.6 \%$ a un $47.02 \%$ que contribuyen a su propia seguridad social en salud, y que la tendencia durante ese tiempo sea que la mitad de la población debe ser asistida con recursos del RC y del Estado, son datos que deberían preocuparnos y buscar opciones para que más colombianos puedan ingresar al SGSSS como contribuyentes.

Tal vez el principal desafío de los procesos de reforma sea el conciliar los requerimientos de eficiencia con los de solidaridad. En el ámbito del financiamiento, la introducción de seguros de riesgos debe ir acompañada con medidas que eviten la selección (discriminación) de riesgos, y sistemas de subsidios que permitan combinar la solidaridad en el financiamiento con contribuciones en función del riesgo. ${ }^{36}$

Muchos acusan del mal estado de la salud en Colombia al SGSSS surgido de la ley 100 de 1993. En vienticinco años de su existencia ha habido intentos por sacarlo de una situación endémica. Mientras se lograba la universalidad, se sacrificaba la eficiencia. Los recursos económicos limitados han tenido que ser destinados para cubrir una gran población, y, en este proceso, mucho dinero se ha perdido en actos de corrupción y mercantilismo, perjudicando a los aportantes y a los no aportantes. Las aseguradoras y sus prestadores han visto la salud, más como un negocio que como un derecho. Hay esfuerzos encaminados a fortalecer la atención en el tercer nivel, descuidando el primero y el segundo, lo que en la práctica lleva a un trato discriminatorio hacia muchos colombianos. "...es claro considerar la crisis de la salud en Colombia como un problema moral que nace de la Reforma de 1993, la cual fue solicitada por organismos financieros internacionales con base en un proyecto de reforma realizado por un agente extraño a nuestra idiosincrasia...". ${ }^{37}$

35 Agudelo, Carlos et al., "El sistema de salud en Colombia: 20 años de logros y problemas", Ciência \& Saúde Coletiva, 16(6), 2011, pp. 2819.

36 Titelman, D., Ensayos sobre el financiamiento de la seguridad social en salud. Los casos de Estados Unidos-Canadá-Argentina-Chile-Colombia, México, Fondo de Cultura Económica, 2000, p. 48.

37 Gómez Pineda F. H., "La crisis de la salud en Colombia: un problema moral", Rev. Colomb. Salud. Libre, 11(1), 2016, pp. 48-56. 


\section{CONCLUSIONES}

La universalidad es uno de los principios que regulan la seguridad social en Colombia, y pasados vienticinco años de vigencia del SGSSS podemos afirmar que se alcanzó este logro, pues pasamos de tener un 23.7\% en 1991 a un $94.6 \%$ de población asegurada y un 5.2\% restante con acceso al derecho a la salud a cargo de las entidades territoriales, conforme a datos de 2017.

Hemos logrado la cobertura universal, pero la calidad es deficiente, son muchas las quejas de los usuarios frente al sistema que parece colapsado, y cada vez requiere más recursos públicos y privados para subsistir. Tenemos cobertura universal, pero deficiente.

El SGSSS en Colombia fue concebido de tal manera que la mayoría de sus miembros aportaran, y una minoría fuera subsidiada. Hoy, pasados veinticinco años, son más los subsidiados que los que aportan. Lo anterior crea dos efectos: 1) más recursos del Estado que podrían utilizarse para otras necesidades son usados para cubrir los déficits del sector salud, y 2) se crea un sistema desigual a la hora de prestar el servicio, donde los sujetos del sistema EPS e IPS establecen tratos diferenciales entre ellos y los usuarios, dependiendo de la EPS a la que pertenece el paciente y si ésta cuenta o no con el respaldo económico que briden confianza a las IPS a la hora de prestar el servicio; de lo contrario, éste será negado. La crisis del sector se evidencia en el número de tutelas que instauran sus usuarios. Para 2018, una de cada tres tutelas presentadas en el país fueron contra sujetos que hacen parte del SGSSS.

Tenemos más personas afiliadas, pero no todas tienen acceso efectivo a la salud. El país sigue siendo increíblemente desigual a la hora de acceder a centros hospitalarios y profesionales de la salud. Para 2018 tenemos veintitrés de los mejores 58 hospitales de Latinoamérica, pero están concentrados en Bogotá, Medellín, Cali, Bucaramanga, mientras que un número importante de colombianos que se encuentran en regiones como la Orinoquia, Pacífica o Amazonia no tienen acceso ni siquiera a un centro de salud básico. En materia de salud, el país está dividido en castas, los que contribuyen y los subsidiados, los ricos y los pobres, los que viven en zonas donde hay buenos hospitales y el resto.

El SGSSS colombiano lleva mucho tiempo en cuidados intensivos; se han hecho esfuerzos, pero no lo suficientes para salvar al enfermo. El Estado colombiano (especialmente el Congreso de la República y el gobierno nacional) ha buscado salvar a la salud de su grave enfermedad con pañitos de agua tibia, 
y mientras tanto los grandes perjudicados son aquellos que tienen menos recursos, ya sean del régimen subsidiado, pero también del contributivo. Tenemos un sistema universal, pero ineficiente.

\section{BIBLIOGRAFÍA}

Agudelo, Carlos et al., "El sistema de salud en Colombia: 20 años de logros y problemas", Ciência \& Saúde Coletiva, 16(6), 2011.

Álvarez, Gabriel, "Crisis de la salud en Colombia: limitantes del acceso al derecho fundamental a la salud de los adultos mayores", Revista CES Derecho, vol. 7, núm. 2, julio-diciembre de 2016.

AYALA, Jhorland, "La salud en Colombia: más cobertura, pero menos acceso", Documentos de Trabajo sobre la Economía Regional, núm. 204, Bogotá, Banco de la República, julio de 2014.

DUQUE QuiNTERO, S. et al., "La seguridad social como un derecho fundamental para las comunidades rurales en Colombia. Opinión jurídica”, 16(32), enero de 2018, disponible en: https://doi.org/https://doi.org/10.22395/ ojum.v16n32a8.

Gómez PinedA, F. H., "La crisis de la salud en Colombia: un problema moral”, Rev. Colomb. Salud. Libre, 2016, 11(1).

GÓMEZ-ARIAS, R., "Colombia: ¿qué ha pasado con su reforma de salud?", Rev. perú. med. exp. salud publica, Lima, vol. 31, núm. 4, octubre-diciembre de 2014.

INFORME DE LA COMISIÓN DEL GASTO Y LA INVERSIÓN PÚBLICA, febrero de 2017, disponible en: http://mmw.minhacienda.gov.co/HomeMinhacienda/ ShowProperty?nodeId=/OCS/P_MHCP_WCC-096783//idcPrimaryFile\&re vision=latestreleased.

INFORME DE LA COMISIÓN DEL GASTO Y LA INVERSIÓN PÚBLICA, febrero de 2017, disponible en: http://mmm.minhacienda.gov.co/HomeMinhaciendal ShowProperty?nodeId=/OCS /P_MHCP_WCC-096783//idcPrimaryFile\&re vision = latestreleased.

MendietA, David y ToBón, M. L. (2018a), "La dignidad humana y el Estado social y democrático de derecho: el caso colombiano", Revista de Estudos Constitucionais, Hermenêtica e Teoria do Direito (RECHTD), 10(3), septiembre-diciembre de 2018. 
MENDIETA, David, "El (des)control de constitucionalidad en Colombia", Estudios Constitucionales, año 16, núm. 2, 2018.

MENDiZÁBAL, G., "Apuntes sobre el derecho internacional de la seguridad social y su relación con américa latina", Revista Latinoamericana de Derecho Social, núm. 25, julio-diciembre de 2017.

Ministerio de SAlud y Protección Social. Dirección de EPIDEMiOLOGÍA Y DEMOGRAFÍA, Guía conceptual y metodológica para la caracterización de la población afiliada a las Entidades Administradoras de Planes de Beneficios de Salud (EAPB), Bogotá, 2017.

Ministerio de Salud y Protección Social, Politica de atención integral en salud, Bogotá, 2016.

OCAMPO Jose Antonio, "Distribución del ingreso, pobreza y gasto social en América Latina”, Revista de la CEPAL, núm. 65, agosto de 1998.

OCAMPO, J. y OSLEY GARZÓN, M., "El sistema de riesgos laborales frente al trabajador del sector informal", Opinión Jurídica, 15(30), diciembre 2016, disponible en: https:// doi.org/ https:// doi.org/10.22395/ojum.v15n30a9.

RiverA, A. F., "Coyuntura del sistema de salud en Colombia: caracterización de una crisis, desde las particularidades financieras de las EPS", Gestión \& Desarrollo, 10 (1), 2013.

SuÁREZ-Rozo L. F., "La crisis del sistema de salud colombiano: una aproximación desde la legitimidad y la regulación", Rev. Gerenc. Polít. Salud, 2017; 16 (32).

Titelman, D., Ensayos sobre el financiamiento de la seguridad social en salud. Los casos de Estados Unidos-Canadá-Argentina-Chile-Colombia, México, Fondo de Cultura Económica, 2000. 